\title{
A new gold-standard medical therapy for CPR in patients with in-hospital cardiac arrest?
}

Therapy with vasopressin, epinephrine, and corticosteroids given during cardiopulmonary resuscitation (CPR) for in-hospital cardiac arrest (IHCA) improves neurologically favourable survival to hospital discharge when compared with epinephrine plus placebo. This finding comes from a multicentre, randomized, controlled trial conducted in Greece. The results "constitute the first evidence for increased vasopressin-steroids-epinephrine (VSE) efficacy ... and are consistent with recently published data casting doubt about epinephrine efficacy ... in achieving longterm survival with favourable neurological recovery," says Dr Spyros Mentzelopoulos, who was lead author of the study report.

During cardiac arrest, endogenous vasopressin and epinephrine are released in the context of the stress response, but plasma levels of vasopressin have been found to be lower among individuals who did not survive a cardiac arrest than in those who do survive. In addition, data from animal studies and preliminary clinical trials indicate that vasopressin improves outcomes after CPR when

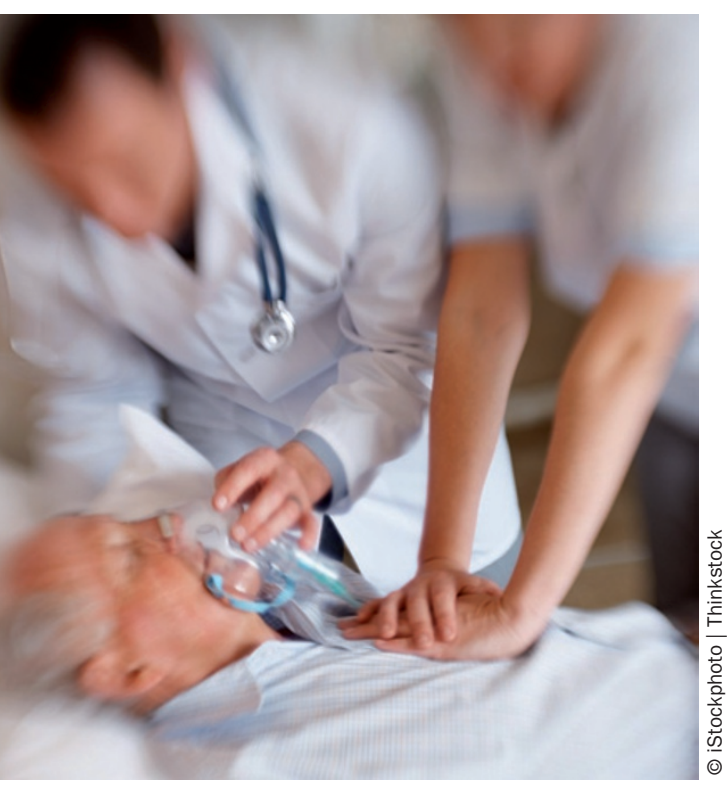

compared with epinephrine alone. The addition of a corticosteroid to vasopressin and epinephrine during $\mathrm{CPR}$, and as monotherapy for postresuscitation shock, is though to improve haemodynamics and attenuate the inflammatory response after cardiac arrest. Methylprednisolone, in particular, is thought to enhance the effects of vasopressin and epinephrine.

Mentzelopoulos et al. consecutively studied 268 patients who experienced an IHCA at one of three tertiary care centres in Greece. Patients who were aged $<18$ years, had a life expectancy of $<6$ weeks, experienced cardiac arrest caused by traumatic blood loss (e.g. aortic rupture), or had 'do not resuscitate' status were not eligible for enrollment in the study. Participants were randomly assigned to receive VSE (arginine vasopressin $20 \mathrm{IU}$ per cycle, plus epinephrine $1 \mathrm{mg}$ per cycle; $n=130$ ) or epinephrine plus a saline placebo $(n=138)$ for the first five cycles of CPR. During the first cycle only, patients in the VSE group were also given methylprednisolone sodium succinate $40 \mathrm{mg}$. Guideline-directed resuscitation protocols were continued if return of spontaneous circulation (ROSC) was not achieved after five cycles of CPR. Patients who developed postresuscitation shock after $4 \mathrm{~h}$ received hydrocortisone $(300 \mathrm{mg}$ per day for $\leq 7$ days and then $200 \mathrm{mg}$ and $100 \mathrm{mg}$ consecutively on the subseqent 2 days; VSE group) or normal saline placebo (controls).

Patients in the VSE group were more likely to achieve ROSC for $\geq 20 \mathrm{~min}$ than those in the control group ( $83.9 \%$ vs $65.9 \%$; OR 2.98, 95\% CI 1.39-6.40, $P=0.005)$. In addition, survival to hospital discharge with favourable neurological recovery (defined as Glasgow-Pittsburgh Cerebral Performance Category 1 or 2) occurred more frequently in the VSE than the control group (13.9\% vs 5.1\%; OR 3.28, 95\% CI 1.17-9.20, $P=0.02)$. The incidence of complications and eventual causes of death among patients who survived $>4$ h were not significantly different between the two groups. Among patients with postresuscitation shock, those in the VSE group were more likely to survive to hospital discharge with favourable neurological recovery than individuals in the control group (21.1\% vs $8.2 \%$; OR 3.74 , 95\% CI 1.20-11.62, $P=0.02)$.

"A complex pathophysiology, such as ... cardiopulmonary arrest cannot be treated with a 'golden bullet', but with a complex strategy, as shown in this paper," comments Professor Volker Wenzel from Innsbruck Medical University, Austria, who was not involved in the study. "This new strategy is simple, inexpensive, and has relatively few side effects ... thus, the risk:benefit ratio is excellent." Professor Gordon Ewy, from the University of Arizona Sarver Heart Center, USA, who was also not involved in the trial, cautions that "these findings, while important, should not be recommended or applied to patients with out-of-hospital cardiac arrest [the aetiologies of which are different from those of IHCA]." However, he suggests that "the combination of epinephrine and vasopressin might be something that should be studied for patients with out-of-hospital cardiac arrest."

Dr Mentzelopoulos' take-home message for clinicians is that "effective haemodynamic support during resuscitation should be complemented by effective support throughout the postresuscitation period; ... both these treatments are [likely to be] necessary for the achievement of improved outcomes". The investigators now plan to study the effects of steroid therapy during and after $\mathrm{CPR}$, and without vasopressin during CPR, in patients with IHCA.

Alexandra Roberts 